BA-TH/01-412

RCG $01 / 10$

gr-qc/0103035

\title{
Detecting a relic background of scalar waves with LIGO
}

\author{
M. Gasperini ${ }^{(1,2)}$ and C. Ungarelli ${ }^{(3)}$ \\ (1) Dipartimento di Fisica, Università di Bari, \\ Via G. Amendola 173, 70126 Bari, Italy \\ (2) Istituto Nazionale di Fisica Nucleare, Sezione di Bari, Bari, Italy \\ (3) Relativity and Cosmology Group \\ School of Computer Science and Mathematics, University of Portsmouth, \\ Portsmouth P01 2EG, England
}

\begin{abstract}
We discuss the possible detection of a stochastic background of massive, nonrelativistic scalar particles, through the cross correlation of the two LIGO interferometers in the initial, enhanced and advanced configuration. If the frequency corresponding to the mass of the scalar field lies in the detector sensitivity band, and the non-relativistic branch of the spectrum gives a significant contribution to energy density required to close the Universe, we find that the scalar background can induce a non-negligible signal, in competition with a possible signal produced by a stochastic background of gravitational radiation.
\end{abstract}




\section{INTRODUCTION}

In the next few years many gravitational antennas will be collecting data, as the new interferometric detectors (GEO, LIGO, TAMA, VIRGO) [1] will join the resonant detectors (ALLEGRO, AURIGA, EXPLORER, NAUTILUS, NIOBE) [2] already in operation, covering a frequency band from $\sim 10 \mathrm{~Hz}$ up to $1 \mathrm{KHz}$. Through the cross-correlation of these antennas it will be possible to search for a stochastic background of primordial gravitational radiation [3], [⿴囗十). The detection of a relic background of gravitational waves would allow us to reconstruct the very early stages of cosmological evolution [5] and even an upper limit would be significant, since there are predictions of very high relic backgrounds [6] in principle detectable already by second generation interferometers [7] (see however [8]).

A generic prediction of unified theories (such as supergravity and superstring theories) is the existence of a gravitational multiplet which includes, beside the usual spin-2 graviton, scalar components. In a cosmological context, as well as the production of a primordial background of gravitational waves, it is therefore worth investigating the possible production of a relic background of scalar waves (for instance, dilatons [9]). In particular, if the mass of the scalar field is small enough $(m \lesssim 100 \mathrm{MeV})$, such a background would be present even today, and it could be accessible to direct experimental observation. It seems thus appropriate to estimate the sensitivity of gravitational antennas also to scalar waves.

A relic background of scalar particles can interact with a gravitational antenna in two ways: either indirectly, through the geodesic coupling of the detector to the induced background of scalar metric fluctuations, or even directly [10], through the effective scalar charge of the detector (such a direct coupling cannot be absorbed into the metric interactions, in an appropriate frame, if the scalar charge is non-universal). Up to now, the sensitivity to scalar waves has been mainly studied using the indirect coupling of the antennas to the scalar part of metric fluctuations, and considering massless scalar fields (see for instance [11]). Under those assumptions, the only difference with respect to the case of standard gravitational radiation is represented by the polarisation tensor of the scalar wave [12].

However, if the detector response tensor is characterised by a symmetric $3 \times 3$ tracefree tensor (such as the differential mode of an interferometer), the sensitivity to a scalar wave of momentum $p$ and energy $E$ generated by a massive scalar field is suppressed by a factor [12] $(p / E)^{4}$ with respect to the sensitivity to an ordinary gravitational wave ${ }^{\text {. This }}$ suppression is ineffective only for modes which are ultrarelativistic in the sensitivity band of the detector, $m \ll E=\left(p^{2}+m^{2}\right)^{1 / 2} \simeq p$; this condition, however, occurs when the frequency corresponding to the mass of the scalar field is much smaller than the typical frequency of the detector, i.e. when $m \ll E_{0} \sim 10-10^{3} \mathrm{~Hz}$. The scalar field would be then associated to longrange interactions, and its coupling to the mass of the detector should be highly suppressed (with respect to the standard gravitational coupling), in order to agree with the existing tests of the equivalence principle and of macroscopic gravitational interactions [13]. Hence, as far as interferometric antennas are concerned, the possible detection of a background of

\footnotetext{
*This factor coincides with the suppression factor for the cross section of a resonant bar to longitudinal massive scalar modes [10].
} 
(massive or massless) scalar waves would seem to be strongly unfavoured with respect to the detection of a graviton background (in the literature, indeed, the possible detection of scalar waves is usually demanded to future resonant detectors of spherical type [14]).

Nevertheless, it is important to notice that, when the mass of the scalar field corresponds to a frequency which is in the detector sensitivity band, it is possible to obtain a resonant response also from the non-relativistic part of the scalar waves spectrum [15]. On the other hand, unlike a relativistic background of massless particles (like gravitons), the nonrelativistic part of a relic background is not constrained by the nucleosynthesis bound, and could saturate the critical energy density required to close the Universe. As suggested in in [15], if the non-relativistic branch of the spectrum is peaked at $p \sim m$, the polarisation suppression factor and the weakness of the scalar coupling could be compensated by the high relic density, and such a background of scalar waves could be a potential source for interferometric detectors.

The aim of this paper is to discuss the possible detection of a relic stochastic background of massive scalar particles with the two LIGO interferometers (including the enhanced and advanced configurations). The paper is organised as follows. In Section $\mathbb{I}$ we recall the general expression for the optimised signal-to-noise ratio (SNR), obtained by cross-correlating two detectors, with respect to a stochastic background of massive scalar waves. In Section [II] we apply this result to the case of the differential mode of an interferometer, taking into account both the geodesic coupling to the scalar part of the metric fluctuations, and the direct coupling to the scalar field. In Section $\mathbb{I \nabla}$ we discuss some examples, and we show in particular that a relic background of non-relativistic scalar particles, whose energy density provides a significant fraction of the critical energy density, can induce a non-negligible signal in the cross-correlation of the LIGO interferometers (in the enhanced and advanced configurations), if the frequency corresponding to the mass of the scalar field is in the sensitivity band of the detectors. The main results of this paper are finally summarised in Section $\nabla$.

\section{SIGNAL-TO-NOISE RATIO}

We will consider a stochastic background of massive scalar particles, described in terms of a scalar field $\phi(\vec{x}, t)$, and characterised by a dimensionless spectrum $\Omega(p)$,

$$
\Omega(p)=\frac{1}{\rho_{c}} \frac{d \rho}{d \ln p}, \quad \rho_{c}=\frac{3 H_{0} M_{P}^{2}}{8 \pi},
$$

where $p$ is the momentum, $\rho$ is the scalar field energy density, $\rho_{c}$ is the critical energy density, $H_{0}$ the present value of the Hubble parameter, and $M_{P}$ the Planck mass. We shall assume that the scalar field is coupled to the mass of the detector with gravitational strength (or weaker), and that the spectrum extends in momentum space from $p_{0}$ to $p_{1}$. The low frequency cut-off $p_{0}$ may be zero (for growing spectra), or the present Hubble scale (for decreasing spectra), while $p_{1}$ is an high-frequency cut-off which depends on the details of the production mechanism.

Our starting point is the expansion of the scalar field in the momentum space,

$$
\phi(t, \vec{x})=\int_{0}^{\infty} d p \int d^{2} \hat{n}\left\{e^{2 \pi i[E(p) t-p \hat{n} \cdot \vec{x}]} \phi(p, \hat{n})+\text { h.c. }\right\}
$$


where $\hat{n}$ is a unit vector specifying the propagation direction, $\vec{p}=\hat{n} p$ is the momentum vector, and the energy $E(p)$ of each mode is

$$
E(p)=\sqrt{p^{2}+(m / 2 \pi)^{2}}
$$

(we are adopting "unconventional" units $h=1$, so that the proper frequency is simply $f=$ $E$ ). The background of scalar waves is assumed to be isotropic, stationary and Gaussian [3] and satisfies the following stochastic conditions

$$
\begin{aligned}
& \langle\phi(p, \hat{n})\rangle=0, \\
& \left\langle\phi(p, \hat{n}) \phi^{*}\left(p^{\prime}, \hat{n}^{\prime}\right)\right\rangle=\delta\left(p-p^{\prime}\right) \delta^{2}\left(\hat{n}-\hat{n}^{\prime}\right) \Phi(p),
\end{aligned}
$$

where, using the explicit definition of $\Omega(p)$,

$$
\Phi(p)=\frac{3 H_{0}^{2} \Omega(p)}{8 \pi^{3} p E^{2}(p)} .
$$

The scalar background induces on the output of the gravitational detector a strain $h_{\phi}(t)$, proportional to the value of the scalar field $\phi\left(t, \vec{x}_{D}\right)$ at the detector position [12],

$$
h_{\phi}(t)=\int_{0}^{\infty} d p \int d^{2} \hat{n}, F_{\phi}(\hat{n})\left\{e^{2 \pi i\left[E(p) t-p \hat{n} \cdot \vec{x}_{D}\right]} \phi(p, \hat{n})+\text { h.c. }\right\}
$$

where $\vec{x}_{D}$ is the position of the detector centre of mass, and $F_{\phi}$ is the antenna pattern. In particular,

$$
F_{\phi}(\hat{n})=q e_{a b} D^{a b}
$$

where $e_{a b}$ is the polarisation tensor of the scalar wave, $D_{a b}$ is the detector response tensor, and $q$ is the effective coupling strength of the scalar field to the detector. The explicit form of $F_{\phi}$ will be discussed in the next section.

The optimal strategy to detect a stochastic background requires the cross-correlation between the output of (at least) two detectors [3], with uncorrelated noises $n_{i}(t), i=1,2$. Given the two outputs over a total observation time $T$,

$$
s_{i}(t)=h_{\phi}^{i}(t)+n_{i}(t), \quad i=1,2,
$$

one constructs a 'signal' $S$,

$$
S=\int_{-T / 2}^{T / 2} d t d t^{\prime} s_{1}(t) s_{2}\left(t^{\prime}\right) Q\left(t-t^{\prime}\right)
$$

where $Q\left(t-t^{\prime}\right)$ is a suitable filter function, usually chosen to optimise the signal-to-noise ratio:

$$
S N R=\langle S\rangle / \Delta S
$$

where $\Delta S^{2}=\left\langle S^{2}\right\rangle-\langle S\rangle^{2}$ is the variance of $S$. In our case, we can compute the mean value $\langle S\rangle$ by using the expansion (2.6) in momentum space, the statistical independence of the 
two noises (i.e. $\left\langle n_{1}(t) n_{2}\left(t^{\prime}\right)\right\rangle=0$ ), and the fact that the noise and the strain are uncorrelated (i.e. $\left\langle n_{i}(t) h_{\phi}^{i}\left(t^{\prime}\right)\right\rangle=0$ ). By assuming that the observation $T$ is much larger than the typical intervals $t-t^{\prime}$ for which $Q \neq 0$, we obtain

$$
\langle S\rangle=\frac{H_{0}^{2}}{5 \pi^{2}} T \operatorname{Re}\left\{\int_{0}^{\infty} d p \frac{\tilde{Q}(E(p)) \Omega(p) \gamma(p)}{p E^{2}(p)}\right\},
$$

where

$$
\tilde{Q}(E(p))=\int_{-\infty}^{\infty} d \tau e^{2 \pi i(E(p) \tau} Q(\tau)
$$

and

$$
\gamma(p)=\frac{15}{4 \pi} \int d^{2} \hat{n} e^{2 \pi i p \hat{n} \cdot\left(\vec{x}_{D 1}-\vec{x}_{D 2}\right)} F_{\phi}^{1}(\hat{n}) F_{\phi}^{2}(\hat{n})
$$

is the so-called overlap reduction function [3], which depends on the relative orientation and location of the two detectors. In (2.13) the normalisation constant has been chosen so that in the massless case - one obtains $\gamma(p)=1$ for two coincident and coaligned interferometers.

Switching to the frequency domain $(E=f, d p / d f=f / p)$, the mean value of $S$ can be written as

$$
\langle S\rangle=\frac{H_{0}^{2}}{5 \pi^{2}} T \operatorname{Re}\left\{\int_{0}^{\infty} d f \frac{\theta(f-\tilde{m}) \tilde{Q}(f) \Omega\left(\sqrt{f^{2}-\tilde{m}^{2}}\right) \gamma\left(\sqrt{f^{2}-\tilde{m}^{2}}\right)}{\left(f^{2}-\tilde{m}^{2}\right) f}\right\},
$$

where $\tilde{m}=m / 2 \pi$, and $\theta$ is the Heaviside step function. To compute the variance we will assume that for each detector the noise is much larger in magnitude than the strain induced by the scalar wave (i.e. $\left.n_{i}(t) \gg h_{\phi}^{i}(t)\right)$. One obtains [3]

$$
\Delta S^{2} \simeq<S^{2}>\simeq \frac{T}{2} \int_{0}^{\infty} d f P_{1}(|f|) P_{2}(|f|)|\tilde{Q}(f)|^{2},
$$

where $P_{i}(|f|)$ is the one-sided noise power spectral density of the $i$-th detector, defined by

$$
\left\langle n_{i}(t) n_{j}\left(t^{\prime}\right)\right\rangle=\frac{\delta_{i j}}{2} \int_{-\infty}^{\infty} d f e^{2 \pi i f\left(t-t^{\prime}\right)} P_{i}(|f|) .
$$

Introducing the following positive semi-definite inner product in the frequency domain,

$$
(a, b) \doteq \operatorname{Re}\left\{\int_{0}^{\infty} a(f) b(f) P_{1}(f) P_{2}(f)\right\}
$$

the signal-to noise ratio can be written as

$$
(S N R)^{2}=2 T\left(\frac{H_{0}^{2}}{5 \pi^{2}}\right)^{2}\left[\frac{(\tilde{Q}, A)^{2}}{(\tilde{Q}, \tilde{Q})}\right]
$$

where

$$
A=\frac{\theta(f-\tilde{m}) \Omega\left(\sqrt{f^{2}-\tilde{m}^{2}}\right) \gamma\left(\sqrt{f^{2}-\tilde{m}^{2}}\right)}{\left(f^{2}-\tilde{m}^{2}\right) f P_{1}(|f|) P_{2}(|f|)}
$$


The above ratio is maximal if $\tilde{Q}$ and $A$ are proportional, i.e. $\tilde{Q}=\lambda A$. With this optimal choice the SNR reads

$$
S N R=\left(\frac{H_{0}^{2}}{5 \pi^{2}}\right) \sqrt{2 T \mathcal{I}}
$$

where

$$
\mathcal{I}=\int_{0}^{\infty} d p \frac{\Omega^{2}(p) \gamma^{2}(p)}{P_{1}\left(\sqrt{p^{2}+\tilde{m}^{2}}\right) P_{2}\left(\sqrt{p^{2}+\tilde{m}^{2}}\right)\left(p^{2}+\tilde{m}^{2}\right)^{3 / 2} p^{3}}
$$

For $\gamma(p)=(d f / d p) \tilde{\gamma}(f)$ our result reduces to expression for the SNR already deduced in 15] (modulo a different normalisation of the overlap function). The scalar nature of the background is encoded into $\gamma(p)$, and will be discussed in the next Section.

\section{ANTENNA PATTERNS AND OVERLAP REDUCTION FUNCTION}

Given the spectrum, and the noise power spectral densities of the two detectors, the computation of the signal-to-noise ratio requires now the explicit expression of the overlap reduction function $\gamma(p)$ for a pair of gravitational antennas. As already pointed out in the Introduction, we shall restrict our analysis to interferometric detectors. We will also consider the interaction of the scalar waves with the differential mode of the interferometer (see e.g. [12]), which is described by the following symmetric, trace-free tensor [16]:

$$
D_{a b}=\frac{1}{2}\left(\hat{u}_{a} \hat{u}_{b}-\hat{v}_{a} \hat{v}_{b}\right), \quad a, b=1,2,3,
$$

where $\hat{u}_{a}, \hat{v}_{a}$ are two unit vectors pointing in the directions of the arms of the interferometer. We will therefore neglect the interaction with the common mode, which is expected to be much more noisy. Note that in this paper we are not interested in distinguishing a scalar signal from a tensor one, but only in estimating the level of the signal eventually induced by a background of scalar waves.

The computation of $\gamma(p)$ requires knowledge of the antenna pattern (2.7), which describes the induced strain and takes into account both the polarisation of the wave and the geometrical configuration of the detector (parametrised by $D_{a b}$ ). As far as the strain induced by a scalar wave is concerned, there are two possible contributions [10]: one corresponds to the direct interaction of the detector with the scalar field, while the other is due to the indirect interaction with the scalar component of the metric fluctuations induced by the scalar field itself.

Indeed, in a general scalar-tensor theory in which the matter fields are non-universally and non-minimally coupled to the scalar field (for instance, gravi-dilaton interactions in a superstring theory context [17]), the macroscopic bodies are characterised by a compositiondependent scalar charge (which cannot be eliminated by an appropriate choice of the frame, like the Jordan frame of conventional Brans-Dicke models), and their motion in a scalartensor background is in general non-geodesic [10]. Taking into account also the direct coupling of a test mass to the gradients of the scalar background field, it follows that the standard 
equation of geodesic deviation (which is the main equation for deriving the response of a gravitational detector) is generalised as follows [10]:

$$
\frac{D^{2} \xi^{\mu}}{D \tau^{2}}+R_{\alpha \beta \nu}^{\mu} u^{\beta} u^{\nu} \xi^{\alpha}+q \xi^{\alpha} \nabla_{\alpha} \nabla^{\mu} \phi=0
$$

Here $\xi^{\mu}$ is the spacelike vector connecting two nearby (non-geodesic) trajectories, and $q$ is the scalar charge per unit of gravitational mass, representing the relative strength of scalar interaction with respect to ordinary tensor-type interactions 10 .

The small, non-relativistic oscillations of a test mass, mechanically equivalent to the detector, are thus described by the equation:

$$
\ddot{\xi}^{a}=-\xi^{b}\left(R_{b 00}^{a}+q \partial_{b} \partial^{a} \phi\right) .
$$

The two contributions to the strain come from the gradients of the scalar field $\phi$, and from the gradients of the scalar component $\psi$ of the metric fluctuations (induced by $\phi$ ), covariantly represented by the Riemann tensor. The two fields $\phi$ and $\psi$ are in principle different, but not independent, being related by a set of coupled differential equations (which are modeldependent). The antenna pattern $F_{\psi}(\hat{n})$, associated to the (indirect) Riemannian part of the strain, has been computed in [12 for both massless and massive scalar fields. We shall see that, for a traceless detector response tensor, the function $F_{\psi}(\hat{n})$ is also proportional to the antenna pattern $F_{\phi}(\hat{n})$ associated to the direct, non-geodesic part of the strain.

Introducing the transverse and longitudinal projectors with respect to the direction of propagation of the scalar wave,

$$
T_{a b}=\left(\delta_{a b}-\hat{n}_{a} \hat{n}_{b}\right), \quad L_{a b}=\hat{n}_{a} \hat{n}_{b}
$$

the indirect, Riemannian contribution to the scalar pattern function becomes [12]:

$$
F_{\psi}(\hat{n})=D^{a b} e_{a b}(\psi)=D^{a b}\left(T_{a b}+\frac{\tilde{m}^{2}}{E^{2}} L_{a b}\right) .
$$

Using eq. (3.3) and the mode expansion for the scalar field, the antenna pattern for the direct, non-geodesic coupling is:

$$
F_{\phi}(\hat{n})=q D^{a b} e_{a b}(\phi)=q D^{a b} \frac{p^{2}}{E^{2}} L_{a b}
$$

Since $T_{a b}=\delta_{a b}-L_{a b}$, and $\operatorname{Tr} D=0$, it follows that

$$
F_{\phi}(\hat{n})=-q F_{\psi}(\hat{n})=-q \frac{p^{2}}{E^{2}} F_{\psi}^{0}(\hat{n})
$$

where $F_{\psi}^{0}$ is the antenna pattern corresponding to a massless scalar wave [12]. The overlap reduction function of two interferometers, directly interacting through a charge $q_{i}$ with a scalar field, can thus be written as

$$
\gamma(p)=q_{1} q_{2}\left(\frac{p}{E}\right)^{4} \gamma_{0}(p)
$$


where $\gamma_{0}(p)$ is the overlap reduction function for the geodesic interaction with a massless scalar field [12]. Using Eq. (2.21) and (3.8), the signal-to-noise ratio is finally given by:

$$
S N R=q_{1} q_{2}\left(\frac{H_{0}^{2}}{5 \pi^{2}}\right)\left[2 T \int_{0}^{\infty} d p \frac{p^{5} \Omega^{2}(p) \gamma_{0}^{2}(p)}{P_{1}\left(\sqrt{p^{2}+\tilde{m}^{2}}\right) P_{2}\left(\sqrt{p^{2}+\tilde{m}^{2}}\right)\left(p^{2}+\tilde{m}^{2}\right)^{11 / 2}}\right]^{1 / 2} .
$$

Note that this expression, with $q_{i}=1$, is also valid to estimate the signal indirectly induced in the interferometers by the scalar metric fluctuations through their usual coupling to the Riemann tensor, provided $\Omega(p)$ refers to the associated spectrum of scalar metric fluctuations. In the following we shall therefore use eq. (3.9) setting $q_{1}=q_{2}=1$ if the dominant signal comes indirectly from the stochastic background of scalar metric fluctuations $\Omega_{\psi}(p)$ induced by the scalar field, and setting instead $q_{i}<1$ (according to the experimental constraints) if the dominant signal is directly due to the stochastic background $\Omega_{\phi}(p)$ of massive scalar waves.

\section{EXAMPLES}

We will now apply the results of the previous sections to estimate the signal induced by a stochastic background due to a massive scalar field on the two LIGO interferometers; we will consider the two detectors operating in the initial (I), enhanced (II) and advanced (III) configurations [18]. In particular, for the noise spectral density we will use the following analytical fits:

- LIGO I 19]:

$$
\begin{aligned}
& P(f)=\frac{3}{2} P_{0}\left[\left(\frac{f}{f_{0}}\right)^{-4}+2+2\left(\frac{f}{f_{0}}\right)^{2}\right], \\
& P_{0}=10^{-46} \mathrm{~Hz}^{-1}, \quad f_{s}=40 \mathrm{~Hz}, \quad f_{0}=200 \mathrm{~Hz} .
\end{aligned}
$$

- LIGO II [20]:

$$
\begin{aligned}
& P(f)=\frac{P_{0}}{11}\left[\left(\frac{f}{f_{0}}\right)^{-9 / 2}+\frac{9}{2}\left(1+\left(\frac{f}{f_{0}}\right)^{2}\right)\right], \\
& P_{0}=7.9 \times 10^{-48} \mathrm{~Hz}^{-1}, \quad f_{s}=25 \mathrm{~Hz}, \quad f_{0}=110 \mathrm{~Hz} .
\end{aligned}
$$

- LIGO III [20]:

$$
\begin{aligned}
& P(f)=\frac{P_{0}}{5}\left[\left(\frac{f}{f_{0}}\right)^{-4}+2+2\left(\frac{f}{f_{0}}\right)^{2}\right], \\
& P_{0}=2.3 \times 10^{-48} \mathrm{~Hz}^{-1}, \quad f_{s}=12 \mathrm{~Hz}, \quad f_{0}=75 \mathrm{~Hz} .
\end{aligned}
$$


In the above equations, $f_{s}$ is a seismic cut-off below which the noise spectral density is treated as infinity. We shall also assume that the non-relativistic modes in the spectrum are the dominant ones, and their energy density almost saturates the critical energy density, namely

$$
\int_{0}^{m} d \ln p \Omega^{\mathrm{non}-\mathrm{rel}}(p) \simeq 1
$$

We shall analyse, in particular, two examples of spectra.

\section{A. Minimal dilaton background}

The first example is a stochastic background of massive relativistic dilatons, produced according to some models of early cosmological evolution based upon string theory [9]. To illustrate the difficulty in detecting such a background, we will consider here the dilaton spectrum obtained in the context of a "minimal" pre-big bang scenario and we shall assume that the dilaton mass is enough small so that the produced dilatons have not yet decayed into radiation with a rate $\Gamma \sim m^{3} / M_{P}^{2}$. This implies $\Gamma \lesssim H_{0}$, i.e $m \lesssim 100 \mathrm{MeV}$.

Even if the mass is negligible at the beginning of the radiation era, the proper momentum $p=k / a(t)$ is red-shifted with respect to the rest mass because of the cosmological expansion, and all the modes in the dilaton spectrum tend to become non-relativistic. As a consequence, the present dilaton spectrum has in general at least three branches, corresponding to: i) relativistic modes, with $p>m$; ii) non-relativistic modes with $p_{m}<p<m$, which became non-relativistic inside the horizon; iii) non-relativistic modes with $p<p_{m}$, which became non-relativistic outside the horizon. The separation scale $p_{m}$ corresponds to a mode that became non-relativistic, $p \simeq m$, just at the time of horizon crossing, $p \simeq H$, and is given by 21] $p_{m}=p_{1}\left(m / H_{1}\right)^{1 / 2}$, where $H_{1}$ is the Hubble scale at the transition between the inflationary phase and the radiation phase, and $p_{1} \simeq H_{1} a_{1} / a \simeq\left(H_{1} / M_{P}\right)^{1 / 2} 10^{11} \mathrm{~Hz}$ is the high frequency cut-off of the spectrum.

The complete dilaton spectrum, at the present time $t_{0}$, can thus be parametrised as follows [22]:

$$
\Omega(p) \simeq \begin{cases}\left(\frac{H_{1}}{M_{p}}\right)^{2} \Omega_{\gamma}(t)\left(\frac{p}{p_{1}}\right)^{\delta}, & m<p<p_{1}, \\ \left(\frac{H_{1}}{M_{p}}\right)^{2}\left(\frac{m^{2}}{H_{1} H_{\mathrm{eq}}}\right)^{1 / 2}\left(\frac{p}{p_{1}}\right)^{\delta-1}, & p_{m}<p<m, \\ \left(\frac{H_{1}}{M_{p}}\right)^{2}\left(\frac{m}{H_{\mathrm{eq}}}\right)^{1 / 2}\left(\frac{p}{p_{1}}\right)^{\delta}, & p<p_{m},\end{cases}
$$

where $\Omega_{\gamma} \sim 10^{-4}$ is the present photon energy density (in critical units), $H_{\text {eq }} \sim 10^{-55} M_{P}$ is the Hubble scale at the epoch of matter-radiation equilibrium, and $\delta \geq 0$ is a slope parameter, depending on the kinematics of the pre-big-bang phase [9] $(\delta=3$ for the low energy dilaton-driven phase, while $\delta<3$ for modes crossing the horizon during the high curvature phase).

Let us recall now that if the dilaton is strongly coupled to macroscopic matter with a composition-dependent charge $q \gtrsim 1$, as suggested by loop corrections to the string effective action [23], then the mass has to be large enough, $m \gtrsim 10^{-4} \mathrm{eV} \sim 10^{11} \mathrm{~Hz}$, to be compatible 
with known phenomenological bounds [24]. In that case, the mass is too far from the sensitivity range of a typical interferometric antenna, and no signal is detectable, since the spectral noises $P_{i}$ are dominated by the mass, and tend to infinity.

To discuss a possible detection, let us thus consider the alternative possibility of (almost) universal string loop corrections discussed in [13], which is compatible with very light (but weakly coupled) dilatons, and let us assume the most favourable situation in which: i) the frequency corresponding to the dilaton mass lies within the sensitivity band of the interferometers; ii) $\delta<1$, and its value is enough small so that the spectrum is dominated by the non-relativistic branch; iii) the spectrum saturates the critical density bound. In such a case, using the explicit form (4.5), it is easy to show that the main contribution to the integral (4.4) comes from the low frequency band $p<p_{m}$, and that the mass and the spectral index are related through the following equation:

$$
\left(H_{1} / M_{P}\right)^{2}\left(m / H_{\mathrm{eq}}\right)^{1 / 2}\left(m / H_{1}\right)^{\delta / 2}=\delta .
$$

With such a small mass, however, the dilatonic interaction is long-range and the direct coupling to matter is strongly suppressed $(q \ll 1$ according to [24]): the SNR turns out to be negligible, as shown by an explicit computation. Let's therefore make the further assumption that the dilaton background induces scalar metric fluctuations with a spectrum which has the same amplitude and the same frequency distribution as the dilaton spectrum (4.5) (this is indeed what happens for relativistic dilatons, at the tree-level, thanks to the gravi-dilaton mixing of the string effective action [9]). Such a background of scalar metric fluctuations would be gravitational coupled to the detectors with $q=1$.

We have computed the signal-to-noise ratio (3.9) obtained by cross-correlating the two LIGO detectors with $q_{1}=q_{2}=1$, using the background (4.5) with $H_{1}$ fixed around the string scale $M_{S} \simeq H_{1}=10^{-1} M_{P}$, and the slope $\delta$ determined by the condition (4.6). We have assumed $T=10^{7} \mathrm{sec}$, and the overlap function $\gamma_{0}(p)$ has been computed using the coordinates of the two LIGO sites (see e.g. 25]).

The results of this computation are summarised in Fig. 1, where the SNR is plotted as a function of the dilaton mass. The scalar signal would be detectable at a good confidence level by LIGO III, for a dilaton mass in the range $20-300 \mathrm{~Hz}$. Unfortunately, however, such values of $m$ compatible with detection would imply for the metric fluctuation spectrum a maximum intensity $\Omega \sim 1$ around a peak scale $p_{m} \sim 10^{-10}-10^{-9} \mathrm{~Hz}$. It follows from eq. (4.5) that $\Omega \gtrsim 10^{-2}$ at $10^{-8} \mathrm{~Hz}$, in sharp contradiction with the bound on metric fluctuations obtained from pulsar-timing data [26] (which requires $\Omega \lesssim 10^{-8}$ at $10^{-8} \mathrm{~Hz}$ ). Imposing the pulsar-timing constraint on the induced spectrum of scalar metric fluctuations it turns out that the signal is not detectable.

\section{B. Non-relativistic scalar dark matter}

The difficulties in detecting the minimal dilaton background discussed in the previous section are mainly due to the fact that the spectrum (4.5) is characterised by a peak located at a scale $p=p_{m}$, much lower than the mass scale $p=m$. However, if the peak of the spectrum is not too far from the mass scale, the signal could be detected even through 
the direct interaction of the scalar background with the interferometers, with an effective coupling compatible with the existing phenomenological bounds.

To illustrate this possibility we shall therefore consider a stochastic background of scalar massive particles whose energy density represents a significant fraction of the critical energy density. We will assume that the spectrum is dominated by non-relativistic modes, with a peak for $p \sim m$. For the sake of simplicity we use the following single-power law for the spectrum:

$$
\Omega(p)=\delta \Omega_{s}\left(\frac{p}{m}\right)^{\delta}, \quad p \leq m, \quad \delta \geq 0,
$$

where the parameter $\Omega_{s}$ is the total energy density (in units of the critical energy density) of the spectrum, integrated over all modes. Since we are interested in a mass for the scalar field corresponding to a frequency in the range $10-10^{3} \mathrm{~Hz}$, we shall assume that the scalar charge $q$ is consequently suppressed: taking into account all existing phenomenological bounds [24], it turns out that in this frequency range the allowed coupling can be parametrised as

$$
\log q^{2} \lesssim-7+\log (m / 10 \mathrm{~Hz}), \quad 10 \mathrm{~Hz} \lesssim m \lesssim 1 \mathrm{kHz}
$$

for universal scalar interactions, and

$$
\log q^{2} \lesssim-8+\log (m / 10 \mathrm{~Hz}), \quad 10 \mathrm{~Hz} \lesssim m \lesssim 1 \mathrm{kHz}
$$

for composition-dependent interactions.

We have computed the signal-to-noise ratio, obtained by cross-correlating the two LIGO detectors, for the spectrum (4.7) assuming a nearly critical energy density $\left(\Omega_{s}=1\right)$ and the maximal value of $q$ allowed by the bound (4.8). Fig. 2 shows the contour plots of the SNR for $T=10^{7} \mathrm{sec}$, as a function of $\log _{10}(m)$ and of the spectral index $\delta$, for the initial (I), enhanced (II) and advanced (III) LIGO configurations. We have considered the case $\delta \leq 3$, typical of string cosmology models [9]. Already with the enhanced LIGO configuration, the signal produced by this class of backgrounds may have a significant signal-to-noise ratio in the relevant mass range. Similar results can be obtained with a composition dependent scalar charge, provided the values of SNR are rescaled by a factor $10^{-1}$, according to eq. (4.9).

Fig 3 shows the values of $q^{2} \Omega_{s}$ corresponding to $\mathrm{SNR}=1$ for the enhanced and advanced LIGO configurations, as a function of the scalar mass, using the spectrum (4.7) with $\delta=1$ (which is a typical value for the slope of the tensor perturbations spectrum predicted in quintessential inflation models [27]). The interesting result is that, in the absence of any signal, an upper limit on $q^{2} \Omega_{s}$ would imply an indirect bound on the scalar charge stronger than the direct bounds existing at present [24], provided the expected energy density of the scalar background corresponds to a non-negligible fraction of critical energy density.

\section{CONCLUSIONS}

In this paper we have discussed the possible detection of a relic background of massive, non-relativistic scalar particles, through the cross-correlation of two interferometric gravitational antennas. 
Using previous results [10, 12, 15], we have computed the signal-to-noise ratio for a stochastic background of massive scalar waves, taking into account both the scalar and gravitational charge of the detector, i.e. including in the differential mode of the interferometer both the direct coupling to the scalar field spectrum and the coupling to the induced spectrum of scalar metric fluctuations.

We have computed the signal-to-noise ratio obtained by cross-correlating the two LIGO interferometers in the initial, enhanced and advanced configurations, for two particular examples: relic dilatons produced in the context of string cosmology models, and a generic scalar component of dark matter.

If the total energy density of the scalar background is dominated by non-relativistic modes and amounts to a significant fraction of the critical energy density, we have found that the induced signal is certainly non-negligible for the LIGO enhanced and advanced configurations, provided both the mass and the peak of the spectrum lie within the sensitivity band of the detectors, i.e. from $10 \mathrm{~Hz}$ to $1 \mathrm{k} \mathrm{Hz}$. These results hold even if the direct coupling to the detector is strongly suppressed, according the existing bounds on long range scalar interactions.

It is worth pointing out that the differential mode of an interferometer is particularly unfavoured for the detection of non-relativistic scalar waves: since the detector response tensor is traceless, the corresponding pattern function, for non-relativistic modes, is suppressed by a factor $(p / E)^{2} \simeq(p / m)^{2} \ll 1$. This is the reason why, to obtain a detectable signal, we have restricted our discussion to spectra peaked around $p=m$. However, for different detectors (for instance, resonant spheres) the detector response tensor with respect a scalar wave is not traceless, and the suppression factor is absent. It would be interesting to investigate the detectability of a more general class of stochastic backgrounds of massive scalar particles (i.e. where the peak and the mass are not in the same frequency range) through resonant spherical detectors.

The main conclusion of our work, confirming the results of previous studies, is that a stochastic background of light but non-relativistic scalar particles, which provides a significant fraction of critical energy density, could induce a non-negligible signal in future enhanced interferometric detectors (in competition with the signal of a possible stochastic background of gravitational waves), provided the frequency corresponding to the mass of the scalar field lies within the detector sensitivity band. We believe that this possibility should be taken into account in the data analysis development of second-generation interferometric detectors.

\section{ACKNOWLEDGMENTS}

It is a pleasure to thank Bruce Allen, Bruce Bassett, Danilo Babusci, Francesco Fucito, Massimo Giovannini, Michele Maggiore and Gabriele Veneziano for helpful comments and/or discussions. We also thank B. Sathyaprakash for having provided an analytical fit of the power spectral density for the noise of the initial LIGO configuration. 


\section{REFERENCES}

[1] A. Abramovici et al., Science 256, 325 (1992); B. Caron et al., Class. Quantum Grav. 14, 1461 (1997); H. Luck and the GEO600 Team, ibid, 1741 (1997); K. Kawabe and the TAMA collaboration, ibid, 1477 (1997).

[2] W. Hamilton, in Second Edoardo Amaldi Conference on Gravitational waves, eds. E. Coccia et al. (World Scientific, Singapore 1998), p. 115; G. V. Pallottino, ibid, p. 105; G. A. Prodi et al., ibid, p. 148; I. Heng, D. Blair, E. Ivanov and M. Tobar, ibid, p. 127.

[3] B. Allen and J. D. Romano, Phys. Rev. D 59, 102001 (1999).

[4] M. Maggiore, Phys. Rep. 331, 283 (2000).

[5] L. P. Grishchuk and M. Solokhin, Phys. Rev. D 43, 2566 (1991).

[6] M. Gasperini and M. Giovannini, Phys. Lett. B 282, 36 (1992); M. Gasperini and G. Veneziano. Astropart. Phys. 1, 317 (1993); M. Gasperini, in Second Paris Cosmology Colloquium (Observatoire de Paris, June 1994), eds. H. J. de Vega and N. Sanchez (World Scientific, Singapore, 1995), p. 429; R. Brustein, M. Gasperini, M. Giovannini and G. Veneziano, Phys. Lett. B 361, 45 (1995).

[7] B. Allen and R. Brustein, Phys. Rev. D 55, 3260 (1997); C. Ungarelli and A. Vecchio, in Gravitational Waves - Third Edoardo Amaldi Conference, ed. S. Meshkov, (AIP Conference Proceedings 523), 90, (2000); D. Babusci and M. Giovannini, Class. Quant. Grav. 17, 2621 (2000).

[8] C. Cartier, E. J. Copeland and M. Gasperini, gr-qc/0101019.

[9] M. Gasperini, Phys. Lett. B 327, 314 (1994); M. Gasperini and G. Veneziano, Phys. Rev. D 50, 2519 (1994); M. Gasperini, in Proc. of the 12th It. Conference on "General Relativity and Gravitational Physics" (Rome, September 1996), edited by M. Bassan et al. (World Scientific, Singapore, 1997), p. 181.

[10] M. Gasperini, Phys. Lett. B 470, 67 (1999).

[11] M. Bianchi, E. Coccia, C. Colacino, V. Fafone and F. Fucito, Class. Quantum Grav. 13, 2865 (1996); M. Bianchi, M. Brunetti, E. Coccia, F. Fucito and J. A. Lobo, Phys. Rev. D 57, 4525 (1998); M. Brunetti E. Coccia, V. Fafone and F. Fucito, Phys. Rev. D 59, 044027 (1999); M. Shibata, K. Nakao and T. Nakamura, Phys. Rev. D 50, 7304 (1994).

[12] M. Maggiore and A. Nicolis, Phys. Rev. D 62, 024004 (2000).

[13] T. Damour and A. M. Polyakov, Nucl. Phys. B 423, 532 (1994).

[14] W. Johnson and S. Merkowitz, Phys. Rev. Lett. 70, 2367 (1993); S. Merkowitz and W. Johnson, Phys. Rev. D 51, 2456 (1995); P. Astone et al., in Second Edoardo Amaldi Conference on Gravitational waves, eds. E. Coccia et al. (World Scientific, Singapore 1998), p. 551.

[15] M. Gasperini, Phys. Lett. B 477, 242 (2000).

[16] R. Forward, Gen. Rel. Grav. 2, 149 (1971); E. Flanagan, Phys. Rev. D 48, 2389 (1993).

[17] M. B. Green, J. Schwartz and E. Witten, Superstring theory (Cambridge University Press, Cambridge, Ma, 1987).

[18] http://www.ligo.caltech.edu/ ligo2/

[19] B. S. Sathyaprakash, private communication.

[20] B. J. Owen and B. S. Sathyaprakash, Phys. Rev. D 60, 022002 (1999).

[21] M. Gasperini and G. Veneziano, Phys. Rev. D 59, 43503 (1999). 
[22] M. Gasperini, in Proc. of the IX Marcel Grossman Meeting (Rome, July 2000), eds. R. Ruffini et al. (World Scientific, Singapore), gr-qc/0009098.

[23] T. Taylor and G. Veneziano, Phys. Lett. B 213, 450 (1988); I. Antoniadis, in Proc. of the XXXIV Rencontres de Moriond on "Gravitational Waves and Experimental Gravity", (Les Arcs, January 1999).

[24] E. Fischbach and C. Talmadge, Nature 356, 207 (1992).

[25] B. Allen, gr-qc/9607075.

[26] V. Kaspi, J. Taylor and M. Ryba, Ap. J. 428, 713 (1994).

[27] M. Giovannini, Phys. Rev. D 60, 123511 (1999). 


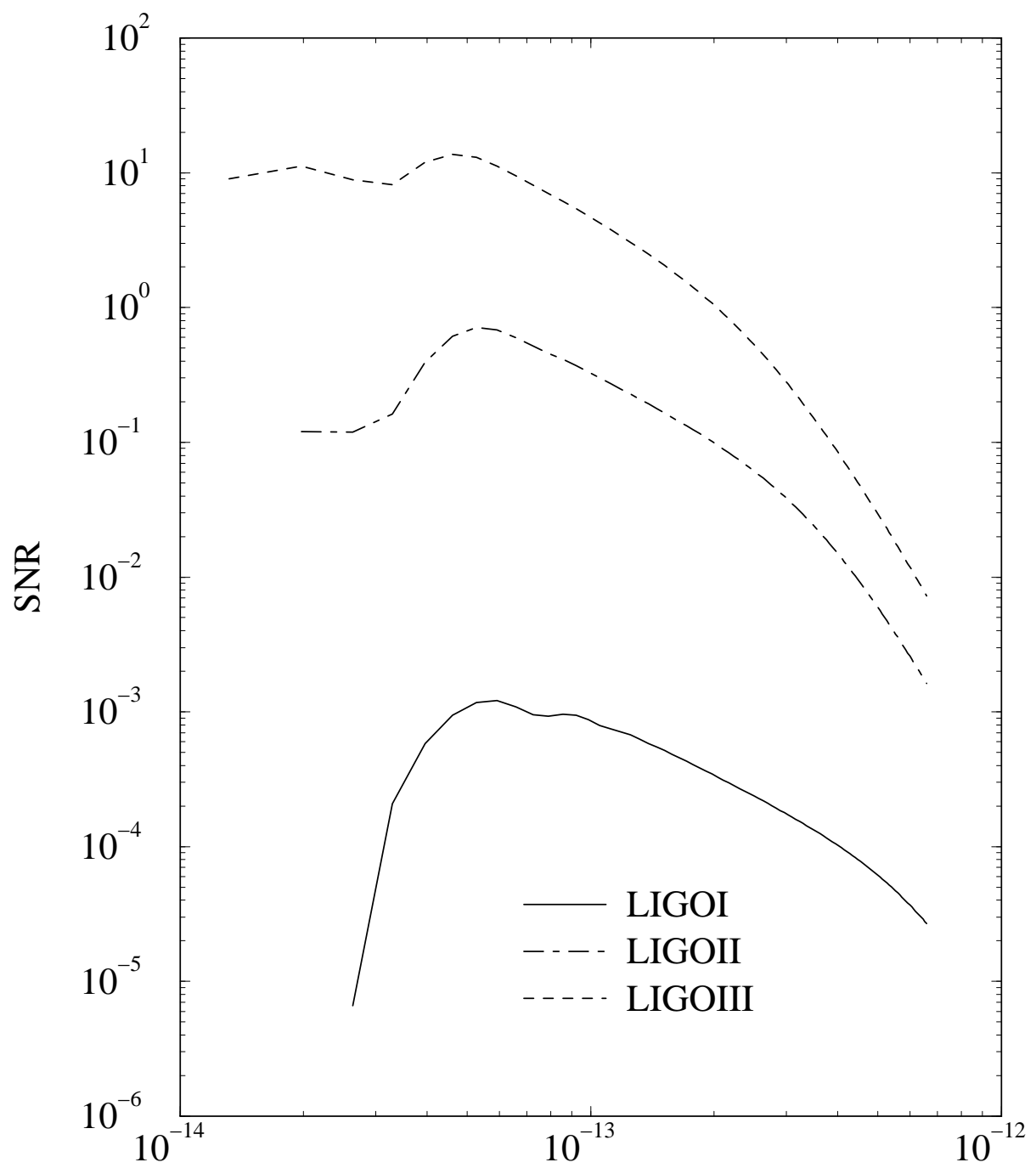

$\mathrm{m}(\mathrm{eV})$

FIG. 1. The signal-to-noise ratio (3.9) for the cross-correlation of the two LIGO interferometers, coupled with maximal strength $\left(q_{1}=q_{2}=1\right)$ to the scalar spectrum (4.5), which is normalised so as to saturate the critical density at $p=p_{m}$. The integration time is $T=10^{7}$ sec. The signal is possibly non-negligible only if the mass is in the sensitivity band of the gravitational detectors. It must be noted, however, that the extrapolation of the spectrum to much lower scales is not compatible with the bounds obtained from pulsar timing data. 

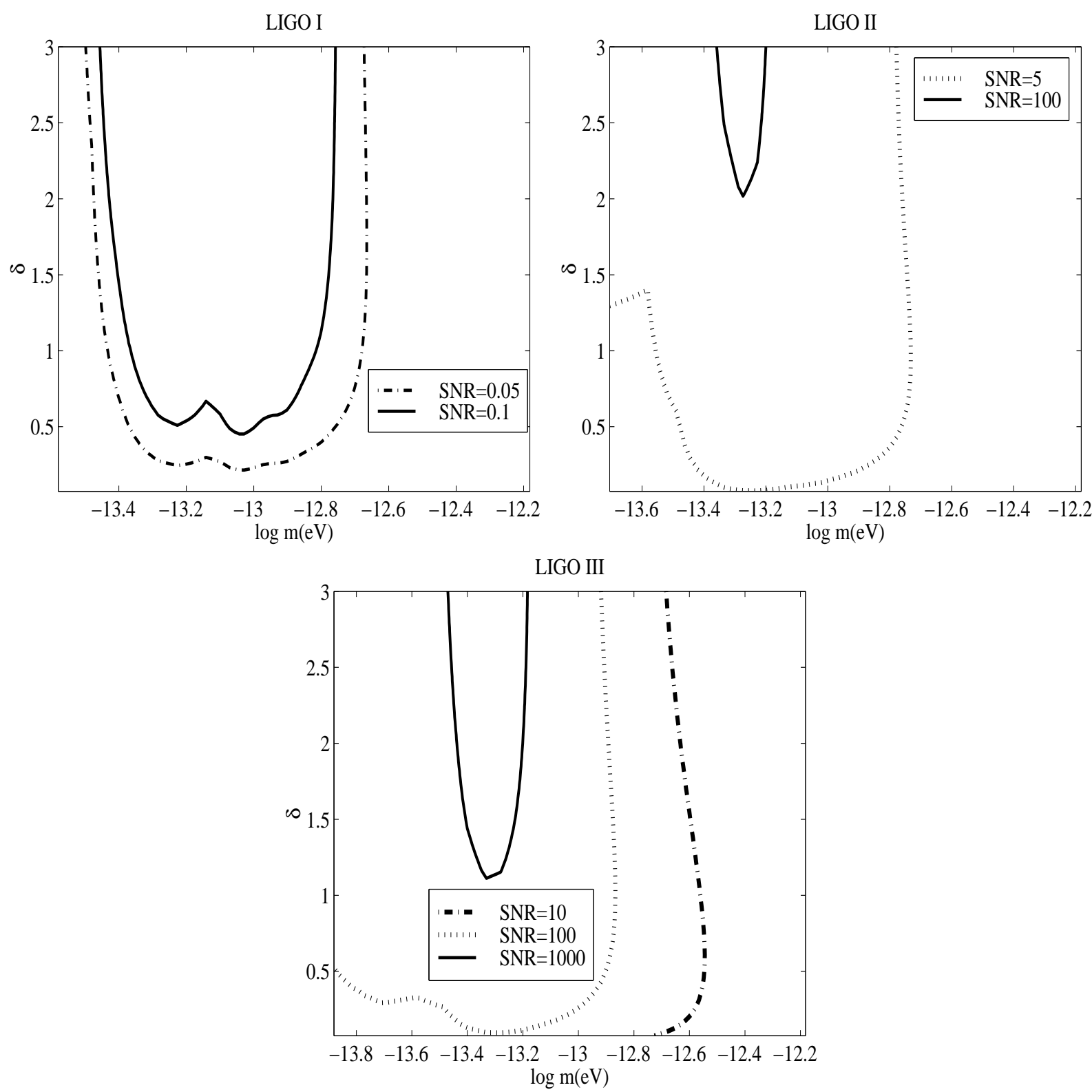

FIG. 2. Contour-plots in the plane $\{m, \delta\}$ for the SNR of eq. (3.9), computed for the scalar spectrum (4.7), and relative to the cross-correlation of a pair of LIGO interferometers in the initial (I), enhanced (II) and advanced (III) configurations. We have used $T=10^{7} \mathrm{sec}, \Omega_{s}=1$, and the maximal allowed value of $q$ according to eq. (4.8). The region inside the contour corresponds to a value of SNR bigger than the value on the border. 


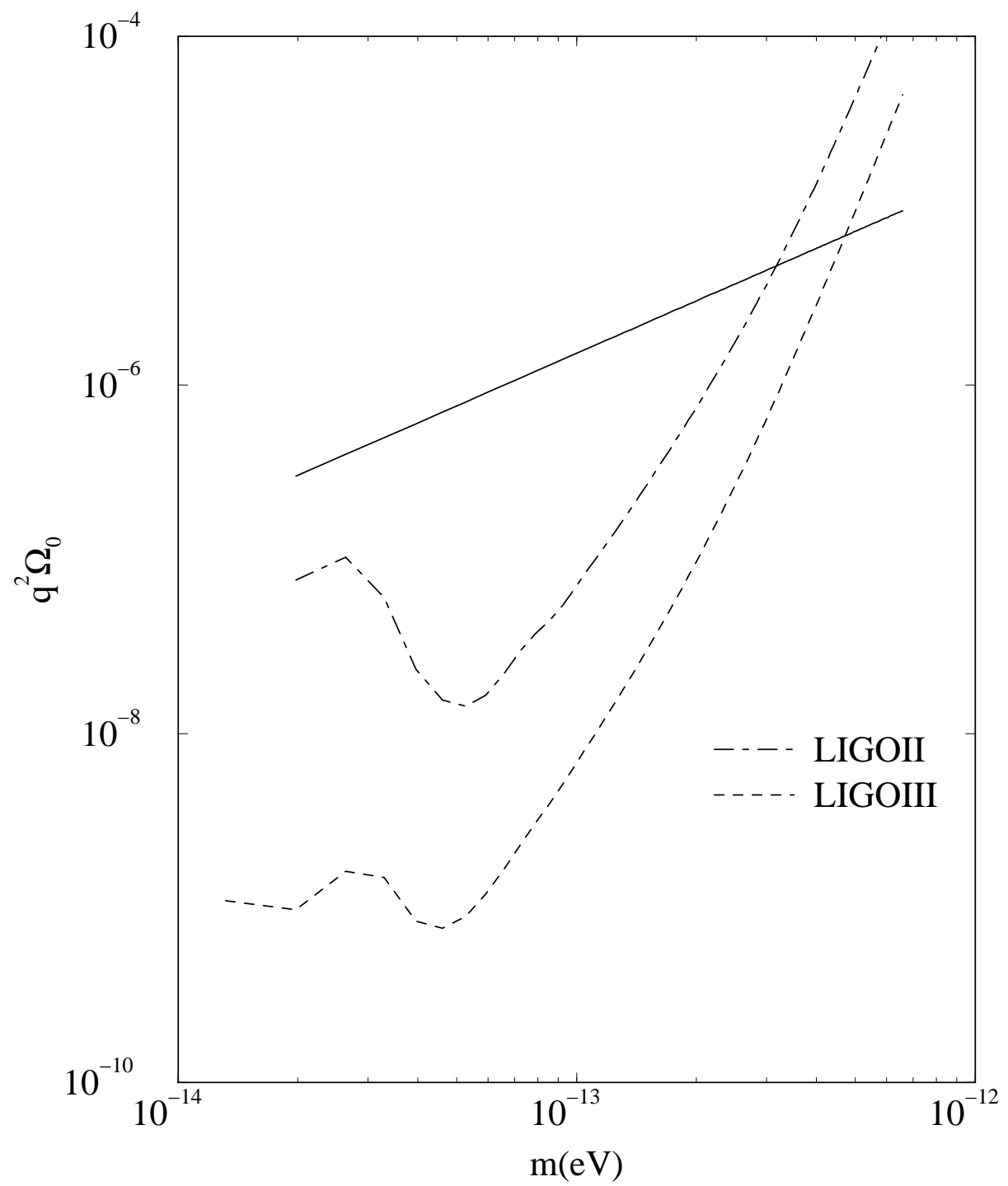

FIG. 3. Allowed values of $q^{2} \Omega_{s}$, as a function of the mass of the scalar field, and corresponding to $S N R=1$, computed for the scalar spectrum (4.7) and for the two LIGO detectors in the enhanced and advanced configurations. For comparison, we have also plotted the maximal value of $q^{2}$ according to eq. (4.8) (solid line). We have used a total observation time $T=10^{7}$ sec, and a spectral index $\delta=1$. 